\title{
Open and hidden charm production in heavy-ion collisions at ultrarelativistic energies
}

\author{
M I Gorenstein ${ }^{1,2}$, A P Kostyuk ${ }^{1,2}$, L McLerran ${ }^{3}$, H Stöcker ${ }^{1}$ \\ and $\mathrm{W}$ Greiner ${ }^{1}$ \\ ${ }^{1}$ Institut für Theoretische Physik, Goethe Universität, Frankfurt am Main, Germany \\ 2 Bogolyubov Institute for Theoretical Physics, Kyiv, Ukraine \\ ${ }^{3}$ Physics Department, Brookhaven National Laboratory, Upton, NY 11979, USA
}

Received 29 November 2001, in final form 21 January 2002

Published 21 June 2002

Online at stacks.iop.org/JPhysG/28/2151

\begin{abstract}
We consider the production of the open charm and $J / \psi$ mesons in heavy-ion collisions at BNL RHIC. We discuss several recently developed pictures for $J / \psi$ production and argue that a measurement at RHIC energies is crucial for disentangling these different descriptions.
\end{abstract}

\section{Introduction}

The production of charmonium states $J / \psi$ and $\psi^{\prime}$ has been measured in nucleus-nucleus $(\mathrm{A}+\mathrm{A})$ collisions at CERN SPS over the last 15 years by the NA38 and NA50 Collaborations. This experimental programme was motivated by a suggestion of Matsui and Satz [1] to use $J / \psi$ as a probe of the state of matter created at the early stage of the collision (see also [2] for a recent review).

In the original picture [1], it is assumed that $J / \psi$ particles are created in primary nucleon-nucleon $(\mathrm{N}+\mathrm{N})$ collisions when the charm-anticharm quark pairs are produced. The fraction (a few per cent) of initial charm pairs which formed a $J / \psi$ state is subsequently reduced in A+A collisions, because of the $J / \psi$ suppression, by a factor which depends upon the details associated with the impact parameter and energy of the collisions.

The statistical approach, proposed in [3], assumes that $J / \psi$ mesons are created at hadronization according to the available hadronic phase-space similar to other (lighter) hadrons. In this case, the $J / \psi$ multiplicity is the same as in a hadron gas (HG) at complete chemical equilibrium. We call this an HG model.

Recently, a picture of the $J / \psi$ creation via coalescence (recombination) of charmed quarks was developed within several model approaches [4-8]. Similar to the HG model [3], the charmonium states are assumed to be formed at the hadronization stage. However, they are produced as a coalescence of earlier created $\mathrm{c}$ and $\overline{\mathrm{c}}$ quarks. Therefore, the $J / \psi$ yield and the total charm yield are connected to each other and differ in general from the HG equilibrium values. 
A principal quantity to be studied in our paper is the ratio of the $J / \psi$ multiplicity to the

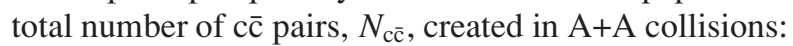

$$
R \equiv \frac{\langle J / \psi\rangle}{N_{\mathrm{c} \overline{\mathrm{c}}}} .
$$

In the standard picture of $J / \psi$ suppression, the number of primary $J / \psi$ particles is proportional to the number of directly produced c $\bar{c}$ pairs with invariant mass below the DD meson threshold [9]. The fraction of the subthreshold pairs in the total number of $N_{\mathrm{c} \bar{c}}$ decreases when the collision energy $\sqrt{s}$ grows. The number of initially produced $J / \psi$ particles is further reduced because of their inelastic interactions with the nucleons from the colliding nuclei. This effect becomes stronger when the number of participants increases. In addition, $J / \psi$ particles can be destroyed by the secondary hadrons (co-movers) created in the A+A collision. The density of these hadrons increases with both the number of nucleon participants $\left(N_{\mathrm{p}}\right)$ and $\sqrt{s}$. The decrease of the ratio $R$ due to the above-mentioned effects is known as 'normal $\mathrm{J} / \psi$ suppression'. The formation of quark-gluon plasma (QGP) at large values of $\sqrt{s}$ and $N_{\mathrm{p}}$ is supposed to lead to a sudden and strong drop of the ratio (1). This is called 'anomalous $J / \psi$ suppression'. Therefore, there is an unambiguous consequence of the standard picture [1, 2]: when the collision energy per nucleon pair $\sqrt{s}$ and/or the number of nucleon participants $N_{\mathrm{p}}$ increase, the ratio (1) decreases.

In models where the $J / \psi$ mesons are produced by recombination of charm quarks at the hadronization stage, the picture can be much different. For the large number of $c \bar{c}$ pairs $\left(N_{\mathrm{c} \overline{\mathrm{c}}} \gg 1\right)$ expected at the RHIC energies, the charmonium states can be formed from a c and

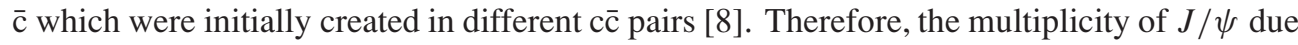
to recombination of charm quarks should be roughly proportional to

$$
\langle J / \psi\rangle \sim \frac{\left(N_{\mathrm{c} \bar{c}}\right)^{2}}{N_{\text {tot }}}
$$

where $N_{\text {tot }}$ is the total multiplicity of produced hadrons. In this case ratio (1) may be constant or even increase (we call this ' $J / \psi$ enhancement') ${ }^{4}$ with $N_{\mathrm{p}}$ and/or $\sqrt{s}$ depending on the underlying physics which generates cē pairs and charmonium states.

We consider the canonical ensemble formulation [6] of the statistical coalescence model (SCM) [5] to describe the $\mathrm{c}$ and $\bar{c}$ recombination into the charmonium states at the hadronization stage. A canonical rather than the more familiar grand canonical formalism is required as we must properly account for the small numbers of charm quarks which are present. When a large number of charm quarks are produced in each collision, these two formalisms become equivalent, but the grand canonical formalism fails for small numbers. As in the standard picture, the charm is created at the very early stage of A+A reaction via hard parton collisions. The $J / \psi$ formation is, however, very different in the standard picture of the $J / \psi$ suppression [1] and in the SCM. This leads to very different relations between the open charm and charmonium yields.

In the analysis below we also present two possibilities of charm chemical equilibration. First, we assume that both the open charm and charmonium yields correspond to a chemically equilibrated hadronic gas. This scenario is the most extreme in its assumptions, but provides a benchmark for the dispersion of various theoretical results. Another possibility is that the number of c̄ pairs, which is an input for the SCM, equals the equilibrium value in the QGP just before its hadronization. These two possibilities are the extremes of scenarios with chemical

4 Please note that our definition of $J / \psi$ enhancement does not involve comparison with proton-proton $(\mathrm{p}+\mathrm{p})$ collisions. We speak about relative $J / \psi$ enhancement in (semi)central A+A collisions, i.e. in more central collisions with respect to less central ones, or at higher $\sqrt{s}$ 's with respect to lower $\sqrt{s}$ 's. It should be stressed that the growing value of the ratio $R$ may remain smaller than the corresponding value of $R$ in $\mathrm{p}+\mathrm{p}$ at the same $\sqrt{s}$. 
equilibration of charm. We do not pretend that these extreme cases really represent what happens in heavy-ion collisions. They are studied to complete our analysis. In fact, the hard parton collisions seem to be the dominant source of the cī pairs created at the RHIC energies.

The issue we want to address in this paper is how to disentangle the different scenarios of charm and charmonium production. It will be shown that the different pictures have a much different dependence on both $N_{\mathrm{p}}$ and $\sqrt{s}$. Distinctive signatures of each of these pictures will be found. We shall argue that $J / \psi$ measurements at RHIC energies, especially in combination with a measurement of open charm, will go far towards resolving the various pictures. Of course, to really disentangle different scenarios, it is useful to be a little more quantitative. That is the central purpose of this paper.

The outline of this paper is as follows. In section 2 we present the equilibrium HG model for the open charm and charmonium production. The picture of the SCM is described in section 3. The results and conclusions of the paper are presented, respectively, in sections 4 and 5.

\section{Hadron gas model}

The equilibrium HG model describes the hadron yields measured in $\mathrm{A}+\mathrm{A}$ collisions in terms of three parameters: volume $V$, temperature $T$ and baryonic chemical potential $\mu_{\mathrm{B}}$. This model seems to work in the AGS-SPS energy region describing successfully not only the pion and (anti)nucleon yields but (multi)strange hadron abundances as well (see, e.g. [10]). For the RHIC energies the temperature parameter $T$ is expected to be similar to that for the SPS energies: $T=175 \pm 10 \mathrm{MeV}$. The baryonic chemical potential becomes small $\left(\mu_{\mathrm{B}}<T\right)$ and decreases with the collision energy.

Does the HG model work for the charm and charmonium yields in A+A collisions? The answer to this question is rather uncertain at present. There are no open charm yield data measured in A+A collisions. This may be a reason why there were no attempts to calculate the open charm hadron yield within the HG model.

\subsection{Grand canonical ensemble treatment of the hadron gas}

The HG model assumes the following formula for the hadron thermal multiplicities in the grand canonical ensemble:

$$
N_{j}=\frac{d_{j} V}{2 \pi^{2}} \int_{0}^{\infty} p^{2} \mathrm{~d} p\left[\exp \left(\frac{\sqrt{p^{2}+m_{j}^{2}}-\mu_{j}}{T}\right) \pm 1\right]^{-1},
$$

where $V$ and $T$ correspond to the volume ${ }^{5}$ and temperature of the HG system, $m_{j}$ and $d_{j}$ denote hadron masses and degeneracy factors. The particle chemical potential $\mu_{j}$ in equation (3) is defined as

$$
\mu_{j}=b_{j} \mu_{\mathrm{B}}+s_{j} \mu_{\mathrm{S}}+c_{j} \mu_{\mathrm{C}},
$$

where $b_{j}, s_{j}$ and $c_{j}$ denote the baryonic number, strangeness and charm of particle $j$. The baryonic chemical potential $\mu_{\mathrm{B}}$ regulates the baryonic density of the HG system whereas strange $\mu_{\mathrm{S}}$ and charm $\mu_{\mathrm{C}}$ chemical potentials should be found from the requirement of zero value for the total strangeness and charm in the system (in our consideration we neglect small effects of a non-zero electrical chemical potential).

5 To avoid further complications we use the ideal HG formulae and neglect excluded volume corrections. 
Some of the particles which are observed after a nuclear collision result from the decay of resonances which have decoupled after the matter fell out of equilibrium. The total multiplicities $N_{j}^{\text {tot }}$ of such observed particles in the HG model are therefore the sum of

$$
N_{j}^{\mathrm{tot}}=N_{j}+\sum_{R} \operatorname{Br}(R \rightarrow j) N_{R}
$$

where $\operatorname{Br}(R \rightarrow j)$ are the corresponding resonance decay branching ratios (both strong and electromagnetic decays are taken into account). The temperatures and chemical potentials used in these relationships are those at decoupling. The hadron yield ratios $N_{j}^{\text {tot }} / N_{i}^{\text {tot }}$ in the grand canonical ensemble are therefore known functions of $T$ and $\mu_{\mathrm{B}}$ variables, and are independent of the volume parameter $V$.

For the thermal multiplicities of both open charm and charmonium states the Bose and Fermi effects are negligible. This is because the charm quark mass is so large that the system is always dilute for charm quark densities. Therefore, equation (3) is simplified to

$$
N_{j}=\frac{d_{j} V \mathrm{e}^{\mu_{j} / T}}{2 \pi^{2}} T m_{j}^{2} K_{2}\left(\frac{m_{j}}{T}\right) \simeq d_{j} V \mathrm{e}^{\mu_{j} / T}\left(\frac{m_{j} T}{2 \pi}\right)^{3 / 2} \exp \left(-\frac{m_{j}}{T}\right),
$$

where $K_{2}$ is the modified Bessel function. The HG model gives the $J / \psi$ yield

$$
N_{J / \psi}^{\text {tot }}=N_{J / \psi}+\operatorname{Br}\left(\psi^{\prime}\right) N_{\psi^{\prime}}+\operatorname{Br}\left(\chi_{1}\right) N_{\chi_{1}}+\operatorname{Br}\left(\chi_{2}\right) N_{\chi_{2}}
$$

where $N_{J / \psi}, N_{\psi^{\prime}}, N_{\chi_{1}}, N_{\chi_{2}}$ are calculated according to equation (6) and $\operatorname{Br}\left(\psi^{\prime}\right) \simeq 0.54$, $\operatorname{Br}\left(\chi_{1}\right) \simeq 0.27, \operatorname{Br}\left(\chi_{2}\right) \simeq 0.14$ are the decay branching ratios of the excited charmonium states to $J / \psi$. Note that $T \simeq 170-180 \mathrm{MeV}$ leads to the $\mathrm{HG}$ value of the thermal ratio of $\left\langle\psi^{\prime}\right\rangle /\langle J / \psi\rangle$

$$
\frac{\left\langle\psi^{\prime}\right\rangle}{\langle J / \psi\rangle}=\left(\frac{m_{\psi^{\prime}}}{m_{J / \psi}}\right)^{3 / 2} \exp \left(-\frac{m_{\psi^{\prime}}-m_{J / \psi}}{T}\right)=0.04-0.05,
$$

in agreement with the data [11] in (semi)central $\left(N_{\mathrm{p}} \gtrsim 100\right.$, which corresponds to the impact parameter range $b \lesssim 8 \mathrm{fm}) \mathrm{Pb}+\mathrm{Pb}$ collisions. This fact was first noticed in [12]. At small $N_{\mathrm{p}}$ as well as in $\mathrm{p}+\mathrm{p}$ and $\mathrm{p}+\mathrm{A}$ collisions the measured value of the $\left\langle\psi^{\prime}\right\rangle /\langle J / \psi\rangle$ ratio is several times larger than its statistical mechanics estimate (8). Therefore, different versions of the statistical approach considered in this paper will be restricted to A+A collisions with $N_{\mathrm{p}}>100$. We do not intend here to describe the open and hidden charm production in $p+p, p+A$ and in very peripheral $\mathrm{A}+\mathrm{A}$ collisions within the statistical models.

Of course, the temperature of decoupling of the hadron gas is quite large, and one should properly be worried about the consistency of such a description. It nevertheless does provide a fair description of the available data on particle production. As such, it provides a standard against which interesting new physics might be calibrated.

\subsection{The canonical ensemble for a hadron gas}

The numbers of charm quarks produced in high-energy nuclear collisions need not be large. Implicit in the grand canonical ensemble treatment is this large-number assumption. The canonical ensemble treatment is valid for any small number of particles, and we now employ it to properly describe charm abundances.

In the canonical ensemble formulation the thermal charmonium multiplicities are still given by equation (6) for a grand canonical ensemble as the charmonium states have zero net charm. The multiplicities given by this equation (6) for open charm hadrons are modified by an additional multiplicative 'canonical suppression' factor. This has been shown, for example, in [13]. The suppression factor is the same for all individual open charm states. Therefore, if 
$N_{\mathrm{O}}$ is the total grand canonical ensemble multiplicity of all open charm and anticharm mesons and (anti)baryons, then the canonical ensemble (c.e.) value of the total open charm is equal to

$$
N_{\mathrm{O}}^{\text {c.e. }}=N_{\mathrm{O}} \frac{I_{1}\left(N_{\mathrm{O}}\right)}{I_{0}\left(N_{\mathrm{O}}\right)},
$$

where $I_{0}, I_{1}$ are the modified Bessel functions. To find $N_{\mathrm{O}}$ we use equation (6) for thermal multiplicities of the open charm hadrons in the grand canonical ensemble and take the summation over all known particles and resonances with open charm [14]. The factor $I_{1}\left(N_{\mathrm{O}}\right) / I_{0}\left(N_{\mathrm{O}}\right)$ in this equation is due to the exact charm conservation. For $N_{\mathrm{O}} \ll 1$ one has $I_{1}\left(N_{\mathrm{O}}\right) / I_{0}\left(N_{\mathrm{O}}\right) \simeq N_{\mathrm{O}} / 2$ and, therefore, the canonical ensemble total open charm multiplicity $N_{\mathrm{O}}^{\text {c.e. }} \sim N_{\mathrm{O}}^{2} \ll N_{\mathrm{O}}$ is strongly suppressed in comparison to the corresponding grand canonical ensemble result. At SPS energies the canonical ensemble suppression effects, due to small numbers of produced charm, are important for the thermal open charm yield even in the most central $\mathrm{Pb}+\mathrm{Pb}$ collisions. These suppression effects become crucial when the number of participants $N_{\mathrm{p}}$ decreases. Note that for $N_{\mathrm{O}} \ll 1$ the multiplicities of the open charm hadrons are proportional to $V^{2}$ in the canonical ensemble $\mathrm{HG}$ formulation (instead of $V$ in the grand canonical ensemble). In the opposite case $N_{\mathrm{O}} \gg 1, I_{1}\left(N_{\mathrm{O}}\right) / I_{0}\left(N_{\mathrm{O}}\right) \rightarrow 1$; therefore the grand canonical ensemble and canonical ensemble results coincide: $N_{\mathrm{O}}^{\text {c.e. }} \rightarrow N_{\mathrm{O}}$. In a high-energy collision of heavy nuclei, the average numbers of produced (anti)baryons, (anti)strange hadrons and charged particles are much larger than 1. Due to this fact, the baryonic number, strangeness and electric charge of the HG system can be treated according to the grand canonical ensemble. This is not true in general for charm.

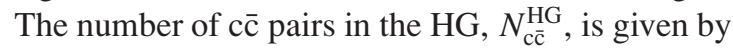

$$
N_{\mathrm{c} \overline{\mathrm{c}}}^{\mathrm{HG}}=\frac{1}{2} N_{\mathrm{O}} \frac{I_{1}\left(N_{\mathrm{O}}\right)}{I_{0}\left(N_{\mathrm{O}}\right)}+N_{\mathrm{H}},
$$

where $N_{\mathrm{H}}$ is the total HG multiplicity of particles with hidden charm.

We can now calculate the $J / \psi$ multiplicity, since this involves only knowing the grand canonical ensemble results for c $\bar{c}$ bound states, and the total open charm multiplicity $N_{\mathrm{O}}^{\text {c.e. }}$, since we now have the canonical ensemble results in hand (9). We need the chemical freeze-out parameters $V, T, \mu_{\mathrm{B}}$ for $\mathrm{A}+\mathrm{A}$ collisions. For the RHIC energies the temperature parameter $T$ is expected to be similar to that for the SPS energies: $T=175 \pm 10 \mathrm{MeV}$. To fix the unknown volume parameter $V$ and baryonic chemical potential $\mu_{\mathrm{B}}$ we use the parametrization of the total pion multiplicity [15]:

$$
\frac{\langle\pi\rangle}{N_{\mathrm{p}}} \simeq C \frac{\left(\sqrt{s}-2 m_{\mathrm{N}}\right)^{3 / 4}}{(\sqrt{s})^{1 / 4}},
$$

where $C \approx 1.46 \mathrm{GeV}^{-1 / 2}$ and $m_{\mathrm{N}}$ is the nucleon mass. Equation (11) is in agreement with both the SPS data and the preliminary RHIC data in Au+Au collisions at $\sqrt{s}=56 \mathrm{GeV}$ and $\sqrt{s}=130 \mathrm{GeV}$. The pion multiplicity (11) should be equated to the total HG pion multiplicity $N_{\pi}^{\text {tot }}$ calculated according to equation (5) which accounts for resonance decays into pionic final states. The HG parameters $V$ and $\mu_{\mathrm{B}}$ are found then as the solution of the following coupled equations:

$$
\begin{aligned}
& \langle\pi\rangle=N_{\pi}^{\mathrm{tot}}\left(V, T, \mu_{\mathrm{B}}\right) \equiv V n_{\pi}^{\mathrm{tot}}\left(T, \mu_{\mathrm{B}}\right) \\
& N_{\mathrm{p}}=V n_{\mathrm{B}}\left(T, \mu_{\mathrm{B}}\right)
\end{aligned}
$$

where $n_{\mathrm{B}}$ is the HG baryonic density. In these calculations we fix the temperature parameter $T$.

The baryonic chemical potential for $\mathrm{Au}+\mathrm{Au}$ collisions at the $\mathrm{RHIC}$ energies is small $\left(\mu_{\mathrm{B}}<T\right)$ and decreases with collision energy. Therefore, most of the thermal HG 
multiplicities become close to their limiting values at $\mu_{\mathrm{B}} \rightarrow 0$. Consequently, most of the hadron ratios $N_{j}^{\text {tot }} / N_{i}^{\text {tot }}$ become independent of the collision energy.

If we assume that the charm quarks reach their chemically equilibrated values, then the chemical potential for charm vanishes. We can then compute the abundances of charmonium. The ratio of $J / \psi$ to negatively charged hadrons is only weakly dependent on the collision energy as a consequence of the considerations of the previous paragraph and is equal approximately to

$$
\frac{N_{J / \psi}^{\text {tot }}}{\left\langle h^{-}\right\rangle} \simeq(0.6-1.5) \times 10^{-6},
$$

for $T=170 \mathrm{MeV}$ and $T=185 \mathrm{MeV}$, respectively. The result (14) is close to the estimate $[3,16]$ of this ratio, $1.0 \times 10^{-6}$, extracted from the NA50 data in $\mathrm{Pb}+\mathrm{Pb}$ collisions at $158 \mathrm{~A} \mathrm{GeV}$.

\subsection{Summary of results}

In this section, we developed the grand canonical ensemble and canonical ensemble formalism for describing abundances of particles in thermal equilibrium. Nucleon and pion abundances were used to constrain the parameters of these treatments: volume and baryon chemical potential. The temperature of the chemical freeze-out was assumed to be equal to its value at

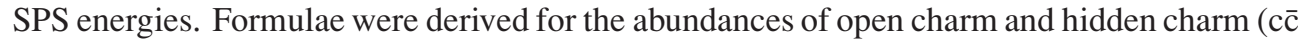
states). In particular, the charm to negative charge particle multiplicity comes out correctly within such a naive treatment.

\section{Statistical coalescence model}

One has serious reservations about the concept of charm chemical equilibration within the $\mathrm{HG}$ approach. The HG system is expected to exist for a much shorter time than is needed for the chemical equilibration of charm. It is more reasonable to assume that the charm quark-antiquark pairs are created at the early stage of $\mathrm{A}+\mathrm{A}$ reaction and the number of $c \overline{\mathrm{c}}$ pairs remains approximately unchanged during the further evolution. After the hadronization, the system would correspond to the HG picture with a non-equilibrium value of the total charm.

We imagine two possible scenarios. In the first, we imagine the ce pairs reach their equilibrium values in the quark-gluon plasma which precedes the hadronic gas. We call this the QGP+SCM model, where the SCM means statistical coalescence model. In the second, we imagine the cic pairs are produced by hard QCD processes early in the collision, and are unchanged by the presence of the hadronic gas or the quark-gluon plasma. This scenario is referred to as the pQCD+SCM model.

\subsection{Charm quarks not in chemical equilibrium}

The SCM [5, 6] assumes that the charmonium states are formed at the hadronization stage. This is similar to the HG model [3]. However, the charmonium states are produced via a

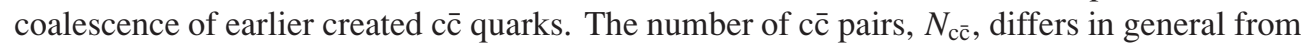
the result expected in the equilibrium HG. One needs then a new parameter $\gamma_{c}$ [5] to adjust the thermal HG results to the required number of $N_{\mathrm{c} \overline{\mathrm{c}}}$ fixed by the early stage evolution. In the grand canonical ensemble, $\gamma_{\mathrm{c}}=\mathrm{e}^{\mu / T}$, where $\mu$ is the chemical potential for $\mathrm{c}+\overline{\mathrm{c}}$ pairs. This is analogous to the introduction of the strangeness suppression factor $\gamma_{\mathrm{s}}[17]$ in the HG model, when the total strangeness observed is smaller than its chemical equilibrium value. 
At high collision energies we find $\gamma_{c}>1$ so that the open charm hadron yield is enhanced by a factor $\gamma_{c}$ and charmonium yield by a factor $\gamma_{c}^{2}$ in comparison with the equilibrium HG predictions. The canonical ensemble formulation of the SCM, as shown in [6], gives

$$
N_{\mathrm{c} \overline{\mathrm{c}}}=\frac{1}{2} \gamma_{\mathrm{c}} N_{\mathrm{O}} \frac{I_{1}\left(\gamma_{\mathrm{c}} N_{\mathrm{O}}\right)}{I_{0}\left(\gamma_{\mathrm{c}} N_{\mathrm{O}}\right)}+\gamma_{\mathrm{c}}^{2} N_{\mathrm{H}} .
$$

Note that the second term in the right-hand side of this equation is proportional to the number of hidden charm states, $N_{\mathrm{H}}$, and is typically very small compared to the total number of charm quarks. It gives only a tiny correction to the first term. Most of the created cē pairs are transformed into the open charm hadrons.

If $N_{\mathrm{c} \overline{\mathrm{c}}} \gg 1$, equation (15) is simplified to [5]: $N_{\mathrm{c} \overline{\mathrm{c}}}=\gamma_{\mathrm{c}} N_{\mathrm{O}} / 2+\gamma_{\mathrm{c}}^{2} N_{\mathrm{H}}$. This happens for central Au+Au collisions at upper RHIC energy $\sqrt{s}=200 \mathrm{GeV}$. For lower RHIC energy $\sqrt{s}=56 \mathrm{GeV}$ the value of $N_{\mathrm{c} \overline{\mathrm{c}}}$ could be close to (or even smaller than) unity so that the canonical ensemble suppression effects for the open charm are still important. Note that for the non-central $\mathrm{A}+\mathrm{A}$ collisions the total charm essentially decreases so that the canonical ensemble suppression effects become stronger, hence their consideration is necessary to study the $N_{\mathrm{p}}$ dependence of the charmonium production even at the upper RHIC energy.

The above equation for the number of c $\bar{c}$ pairs will be used to find the charm enhancement factor $\gamma_{\mathrm{c}}$ and calculate the $J / \psi$ multiplicity:

$$
\langle J / \psi\rangle=\gamma_{\mathrm{c}}^{2} N_{J / \psi}^{\mathrm{tot}},
$$

where $N_{J / \psi}^{\text {tot }}$ is given by equation (7), the equation which accounts for decays of states with hidden charm into the $J / \psi$.

\subsection{Estimating the initial charm production}

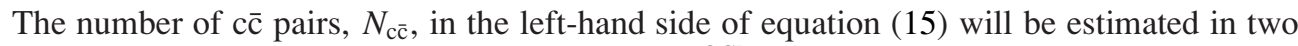
different ways - in the pQCD approach, $N_{\mathrm{c} \overline{\mathrm{c}}}=N_{\mathrm{c} \overline{\mathrm{c}}}^{\mathrm{pQCD}}$, and in the equilibrium QGP just before its hadronization, $N_{\mathrm{c} \overline{\mathrm{c}}}=N_{\mathrm{c} \overline{\mathrm{c}}}^{\mathrm{QG}}$-and then used as the input for the SCM.

The pQCD calculations for $\mathrm{c} \overline{\mathrm{c}}$ production cross section $\sigma(\mathrm{pp} \rightarrow \mathrm{c} \overline{\mathrm{c}})$ in $\mathrm{p}+\mathrm{p}$ collisions were first done in [18]. In [19] the cross section $\sigma(\mathrm{pp} \rightarrow \mathrm{c} \overline{\mathrm{c}})$ was calculated in the next-to-leading order $\mathrm{pQCD}$ approximation and fit to the existing $\mathrm{p}+\mathrm{p}$ data for charm production. We have parametrized the $\sqrt{s}$ dependence of the charm production cross section at $10<\sqrt{s}<200 \mathrm{GeV}$ as

$$
\sigma(\mathrm{pp} \rightarrow \mathrm{c} \overline{\mathbf{c}})=\sigma_{0}\left(1-\frac{M_{0}}{\sqrt{s}}\right)^{\alpha}\left(\frac{\sqrt{s}}{M_{0}}\right)^{\beta},
$$

with $\sigma_{0} \simeq 3.392 \mu \mathrm{b}, M_{0} \simeq 2.984 \mathrm{GeV}, \alpha \simeq 8.185$ and $\beta \simeq 1.132$. For our fit we used the result of [19] obtained with GRV HO set of proton structure functions [20] for charm quark mass and renormalization scale $m_{\mathrm{c}}=\mu=1.3 \mathrm{GeV}$. Formula (17) agrees approximately with the existing data and leads to the value of $\sigma(\mathrm{pp} \rightarrow \mathrm{c} \overline{\mathrm{c}}) \simeq 0.35 \mathrm{mb}$ at $\sqrt{s}=200 \mathrm{GeV}$.

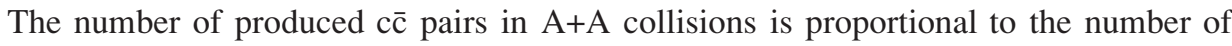
primary nucleon-nucleon collisions:

$$
N_{\mathrm{c} \overline{\mathrm{c}}}^{\mathrm{pQCD}}=N_{\mathrm{coll}}^{\mathrm{AA}}\left(N_{\mathrm{p}}\right) \frac{\sigma(\mathrm{pp} \rightarrow \mathrm{c} \overline{\mathrm{c}})}{\sigma_{\mathrm{NN}}^{\text {inel }}},
$$

where $N_{\text {coll }}^{\mathrm{AA}}\left(N_{\mathrm{p}}\right)$ is the number of primary nucleon-nucleon collisions, which depends on the number of participants and on the details of nuclear geometry and $\sigma_{\mathrm{NN}}^{\text {inel }} \approx 30 \mathrm{mb}$ is the inelastic nucleon-nucleon cross sections. 
For the most central $\mathrm{A}+\mathrm{A}$ collision $\left(N_{\mathrm{p}} \simeq 2 \mathrm{~A}\right)$ the number of primary nucleon collisions can be estimated within the model of homogeneous spherical nucleus [21]:

$$
N_{\mathrm{coll}}^{\mathrm{AA}}\left(N_{\mathrm{p}}\right) \simeq \frac{9}{8} \frac{A^{2}}{\pi R_{A}^{2}} \sigma_{\mathrm{NN}}^{\mathrm{inel}},
$$

with $R_{A} \approx 1.12 A^{1 / 3} \mathrm{fm}$. Therefore $N_{\mathrm{p}}$ dependence on the number of produced ce pairs can be calculated as ${ }^{6}$

$$
N_{\mathrm{c} \overline{\mathrm{c}}}^{\mathrm{pQCD}} \simeq C \sigma(\mathrm{pp} \rightarrow \mathrm{c} \overline{\mathrm{c}}) N_{\mathrm{p}}^{4 / 3},
$$

where $C \approx 11$ barn $^{-1}$.

\subsection{Initial charm from a chemically equilibrated $Q G P$}

We also make the SCM model calculations with $N_{\mathrm{c} \overline{\mathrm{c}}}=N_{\mathrm{c} \overline{\mathrm{c}}}^{\mathrm{QGP}}$ on the left-hand side of equation (15). The values of $N_{\mathrm{c} \overline{\mathrm{c}}}^{\mathrm{QGP}}$ in central Au+Au collisions at different $\sqrt{s}$ are calculated as the equilibrium number of $c \bar{c}$ pairs in the QGP just before its hadronization. The hadronization temperature of the QGP is taken to be equal to the chemical freeze-out temperature in the $\mathrm{HG}$, i.e. we assume that the chemical freeze-out in the HG takes place just after the QGP hadronization. The volume $V_{\mathrm{QGP}}$ of the QGP is found then from the requirement of an approximate entropy conservation during the hadronization transition: $S_{\mathrm{QGP}}=S_{\mathrm{HG}}$ (the non-zero but small baryonic chemical potentials in the HG and in the QGP do not affect the QGP volume estimate). The calculation of $N_{\mathrm{c} \overline{\mathrm{c}}}^{\mathrm{QGP}}$ corresponds to the equilibrium ideal gas of quarks and gluons $\left(m_{\mathrm{u}} \approx m_{\mathrm{d}} \approx 0, m_{\mathrm{s}} \approx 0.12 \mathrm{GeV}, m_{\mathrm{c}} \approx 1.3 \mathrm{GeV}\right)$. In the grand canonical ensemble, one finds for the number of $\mathrm{c}$ and $\overline{\mathrm{c}}$

$$
N_{\mathrm{c}}=N_{\overline{\mathrm{c}}}=\frac{d_{\mathrm{c}} V_{\mathrm{QGP}}}{2 \pi^{2}} \operatorname{Tm}_{\mathrm{c}}^{2} K_{2}\left(\frac{m_{\mathrm{c}}}{T}\right),
$$

where $d_{\mathrm{c}}=6$ is the degeneracy factor for the c quark. Taking into account the exact charm conservation we obtain the number of cē pairs in the canonical ensemble:

$$
N_{\mathrm{c} \overline{\mathrm{c}}}^{\mathrm{QGP}}=\frac{1}{2}\left(N_{\mathrm{c}}+N_{\overline{\mathrm{c}}}\right) \frac{I_{1}\left(N_{\mathrm{c}}+N_{\overline{\mathrm{c}}}\right)}{I_{0}\left(N_{\mathrm{c}}+N_{\overline{\mathrm{c}}}\right)} .
$$

\section{Comparison of various mechanisms}

In this section we present the results of the SCM and the HG model. Two different inputs for $N_{\mathrm{c} \overline{\mathrm{c}}}$ in the SCM (15) are studied: $N_{\mathrm{c} \overline{\mathrm{c}}}^{\mathrm{pQD}}(20)$ and $N_{\mathrm{c} \overline{\mathrm{c}}}^{\mathrm{QG}}(22)$. We call these model formulations as $\mathrm{pQCD}+\mathrm{SCM}$ and QGP+SCM, respectively. The HG model, discussed in section 2, can be considered as a special case of the SCM when $\gamma_{\mathrm{c}} \equiv 1$. First, we consider central Au+Au collisions $\left(N_{\mathrm{p}} \simeq 2 A\right)$ at different collision energies $\sqrt{s}$. Keeping the temperature parameter $T$ fixed we solve equations (12) and (13) to find the hadronization parameters $V$ and $\mu_{\mathrm{B}}$. Equation (11) is used as the parametrization of the $\sqrt{s}$ dependence for the total pion multiplicity. With HG parameters $T, V$ and $\mu_{\mathrm{B}}$ we calculate the thermal multiplicities of individual open and hidden charm states in the grand canonical ensemble according to equation (6) and then find the grand canonical ensemble total open charm $N_{\mathrm{O}}$ and the total hidden charm $N_{\mathrm{H}}$ multiplicities taking the summation over all known open and hidden charm states. Using the calculated values of $N_{\mathrm{O}}$ and $N_{\mathrm{H}}$ we proceed further with three different model formulations along the scheme presented in table 1.

\footnotetext{
6 Strictly speaking, equation (20) does not exactly correspond to the real experimental situation. Au+Au collisions with different centrality rather than central collisions of nuclei with different atomic numbers will be studied at RHIC. Still, formula (20) gives almost the same numerical result as the more realistic calculations [22].
} 


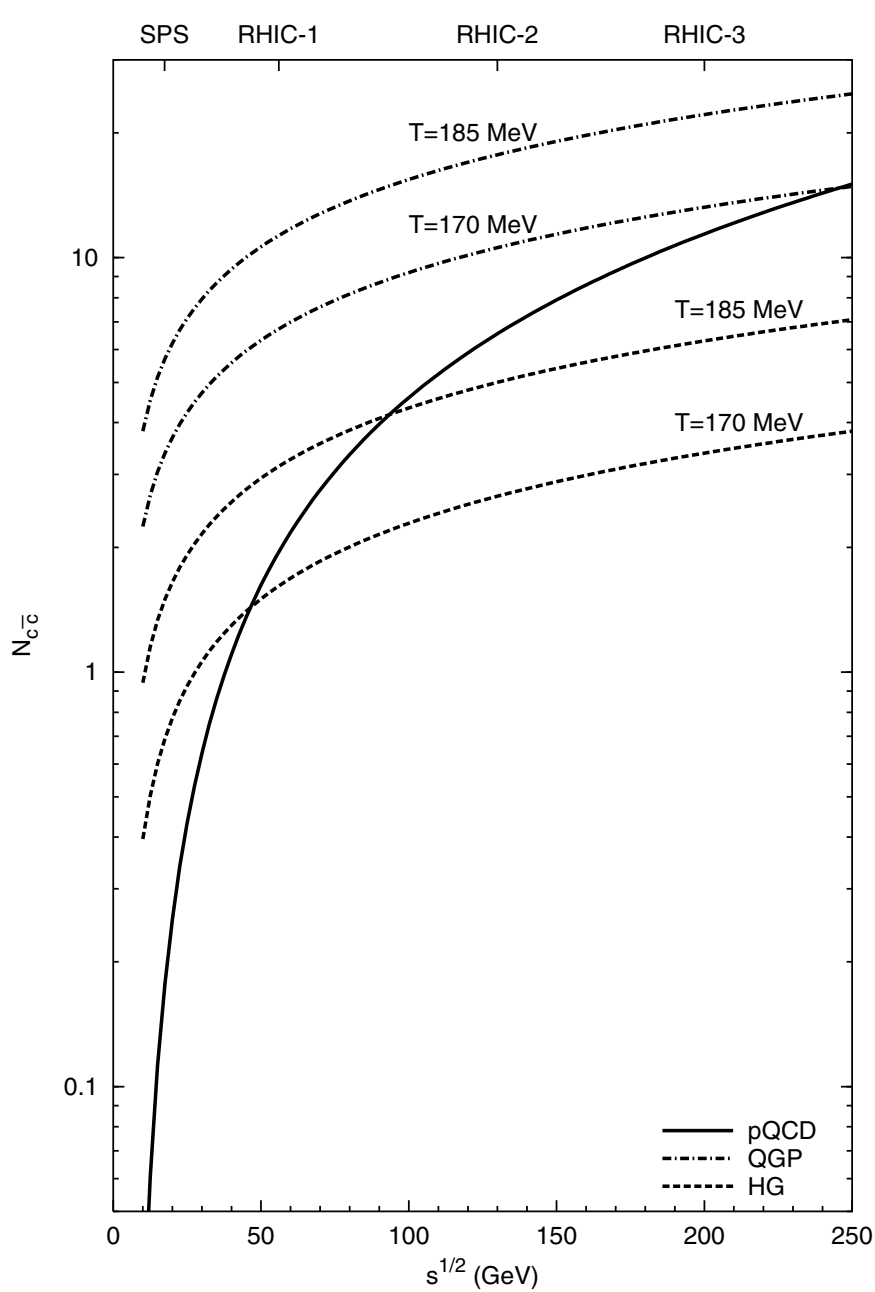

Figure 1. The dependence of the average number of cc pairs per Au+Au central collision on the centre-of-mass energy of the nucleon pair. The calculations are done in the framework of three different models: perturbative QCD (pQCD), equilibrium quark-gluon plasma (QGP) and equilibrium hadron gas (HG). The QGP and HG results are shown for two different temperatures: $T=170 \mathrm{MeV}$ and $T=185 \mathrm{MeV}$.

Table 1. Summary of the models.

\begin{tabular}{llll}
\hline & $\mathrm{pQCD}+\mathrm{SCM}$ & $\mathrm{QGP}+\mathrm{SCM}$ & $\mathrm{HG}$ \\
\hline$N_{\mathrm{c} \overline{\mathrm{c}}}$ & $N_{\mathrm{c} \overline{\mathrm{c}}}^{\mathrm{pQCD}}$, equation $(20)$ & $N_{\mathrm{c} \overline{\mathrm{c}}}^{\mathrm{QGP}}$, equation $(20)$ & $N_{\mathrm{c} \overline{\mathrm{c}}}^{\mathrm{HG}}$, equation $(10)$ \\
$\gamma_{\mathrm{c}}$ & Equation (15), $N_{\mathrm{c} \overline{\mathrm{c}}}=N_{\mathrm{c} \overline{\mathrm{c}}}^{\mathrm{pQCD}}$ & Equation $(15), N_{\mathrm{c} \overline{\mathrm{c}}}=N_{\mathrm{c} \overline{\mathrm{c}}}^{\mathrm{QGP}}$ & $\gamma_{\mathrm{c}} \equiv 1$ \\
$\langle J / \psi\rangle$ & $\gamma_{\mathrm{c}}^{2} N_{J / \psi}^{\mathrm{tot}}$, equation $(16)$ & $\gamma_{\mathrm{c}}^{2} N_{J / \psi}^{\mathrm{tot}}$, equation $(16)$ & $N_{J / \psi}^{\text {tot }}$, equation $(7)$ \\
\hline
\end{tabular}

\subsection{Number of cic pairs as function of energy}

The dependence of the number of c $\overline{\mathrm{c}}$ pairs, $N_{\mathrm{c} \overline{\mathrm{c}}}$, on the collision energy is shown in figure 1 for different model formulations. The value of $N_{\mathrm{c} \bar{c}}^{\mathrm{pQCD}}$ (20) increases strongly with collision energy and at the highest RHIC energy it becomes several times larger than the thermal equilibrium value $N_{\mathrm{c} \bar{c}}^{\mathrm{HG}}$ in the HG model (10). At the SPS energies $(\sqrt{s} \approx 20 \mathrm{GEV})$ the 


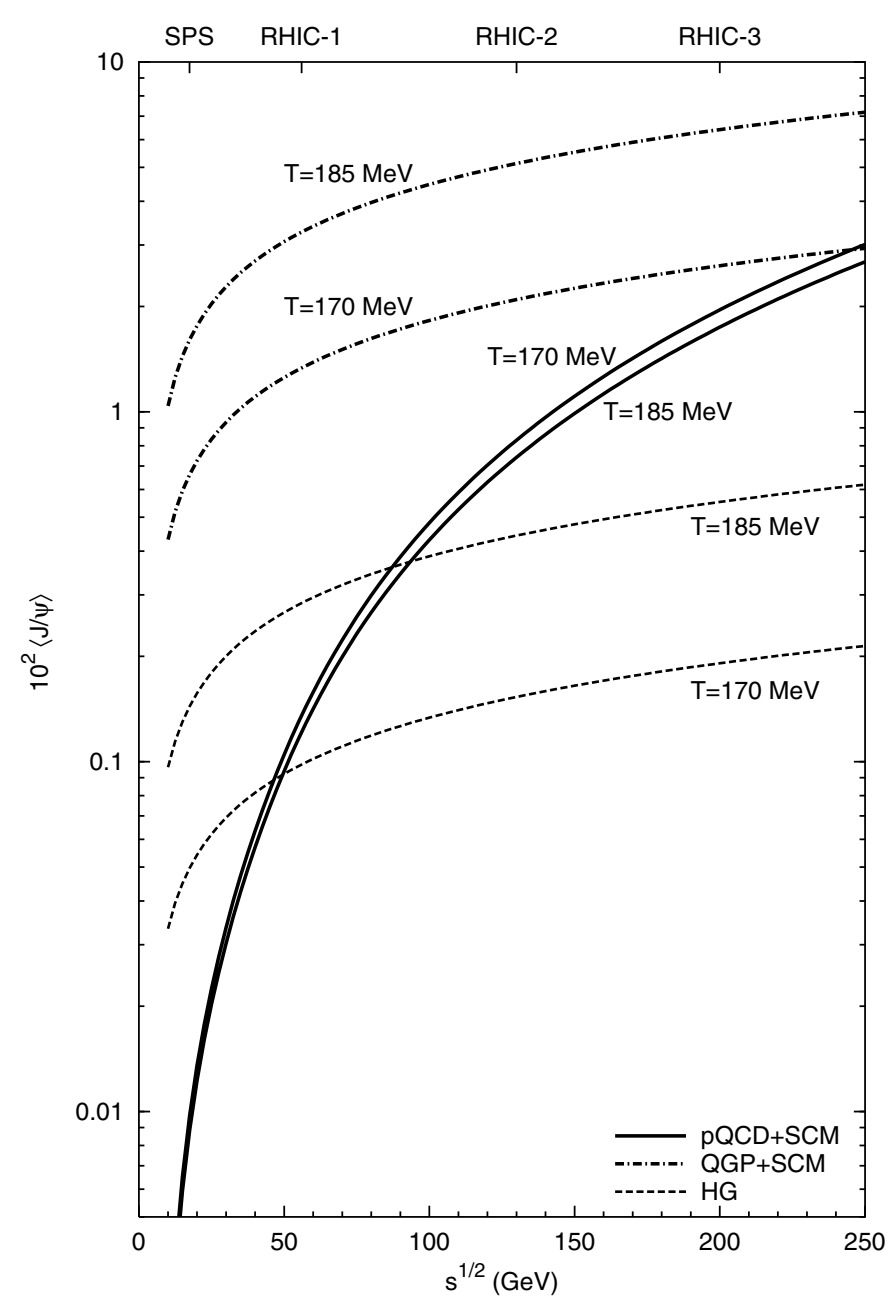

Figure 2. The dependence of the average number of $J / \psi$ particles per Au+Au central collision on the centre-of-mass energy of the nucleon pair. The calculations are done in the framework

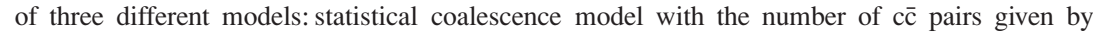
perturbative QCD (pQCD+SCM), statistical coalescence model with the number of cī pairs given by equilibrium quark-gluon plasma (QGP+SCM) and equilibrium hadron gas model (HG). All the dependences are shown for two different temperatures: $T=170 \mathrm{MeV}$ and $T=185 \mathrm{MeV}$.

situation is however different: $N_{\mathrm{c} \overline{\mathrm{c}}}^{\mathrm{HG}}>N_{\mathrm{c} \overline{\mathrm{c}}}^{\mathrm{pCD}}$. The value of $N_{\mathrm{c} \overline{\mathrm{c}}}^{\mathrm{QGP}}$ defined by equation (22) seems to give an upper limit of the charm production in $\mathrm{A}+\mathrm{A}$ collisions. The inequality $N_{\mathrm{c} \overline{\mathrm{c}}}^{\mathrm{QGP}} \gg N_{\mathrm{c} \overline{\mathrm{c}}}^{\mathrm{HG}}$ is mainly because of a small mass of charm quark, $m_{\mathrm{c}} \simeq 1.3 \mathrm{GeV}$, in comparison to the masses of charmed hadrons $\left(\mathrm{D}, \mathrm{D}^{*}\right.$, etc $)$.

\section{2. $\mathrm{J} / \psi$ multiplicity versus energy}

The $J / \psi$ multiplicity is shown in figure 2 and the ratio of $J / \psi$ to negative hadrons in figure 3. The experimental estimate of the $J / \psi$ to $h^{-}$ratio extracted from the NA50 data in $\mathrm{Pb}+\mathrm{Pb}$ collisions at $158 \mathrm{~A} \mathrm{GeV}$ is also presented in figure 3. It seems to be in agreement with the simple $\mathrm{HG}$ results [3] (i.e. $\gamma_{\mathrm{c}} \approx 1$ ). In the pQCD+SCM model the enhancement of 


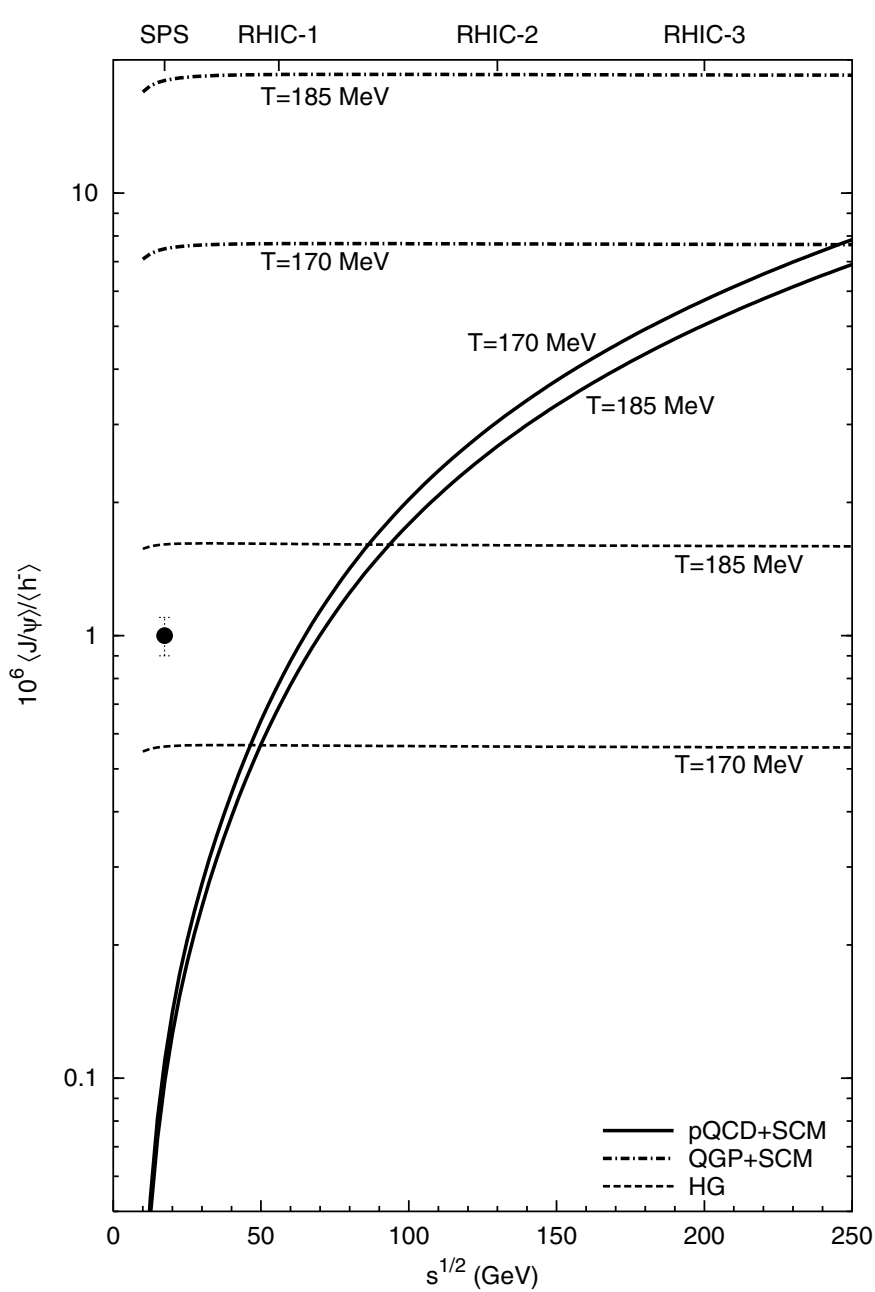

Figure 3. The dependence of the ratio of the $J / \psi$-multiplicity to the negative hadron multiplicity in $\mathrm{Au}+\mathrm{Au}$ central collisions on the centre-of-mass energy of the nucleon pair. The calculations are done in the framework of three different models: statistical coalescence model with the number of c $\bar{c}$ pairs given by perturbative QCD (pQCD+SCM), statistical coalescence model with the number

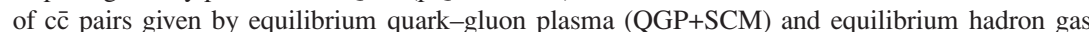
model (HG). All the dependences are shown for two different temperatures: $T=170 \mathrm{MeV}$ and $T=185 \mathrm{MeV}$. The filled circle shows the value estimated from the NA50 experimental data for $\mathrm{Pb}+\mathrm{Pb}$ collisions at the SPS energy $[2,14]$.

the total charm $N_{\mathrm{c} \overline{\mathrm{c}}}$ by a factor of about 3 is needed to explain the observed $J / \psi$ multiplicity [6]. This prediction of the statistical coalescence model can be tested in the near future (measurements of the open charm are planned at CERN). Such a comparison will also require to specify more accurately the $\langle J / \psi\rangle$ data. A possible source of the open charm enhancement in $A+A$ collisions at the SPS with respect to the direct extrapolation (20) from $p+p$ to $\mathrm{A}+\mathrm{A}$ may be the broadening of the phase space available for the open charm production due to the presence of the quark-gluon medium [23]. This effect, however, is expected to be small at RHIC energies. 


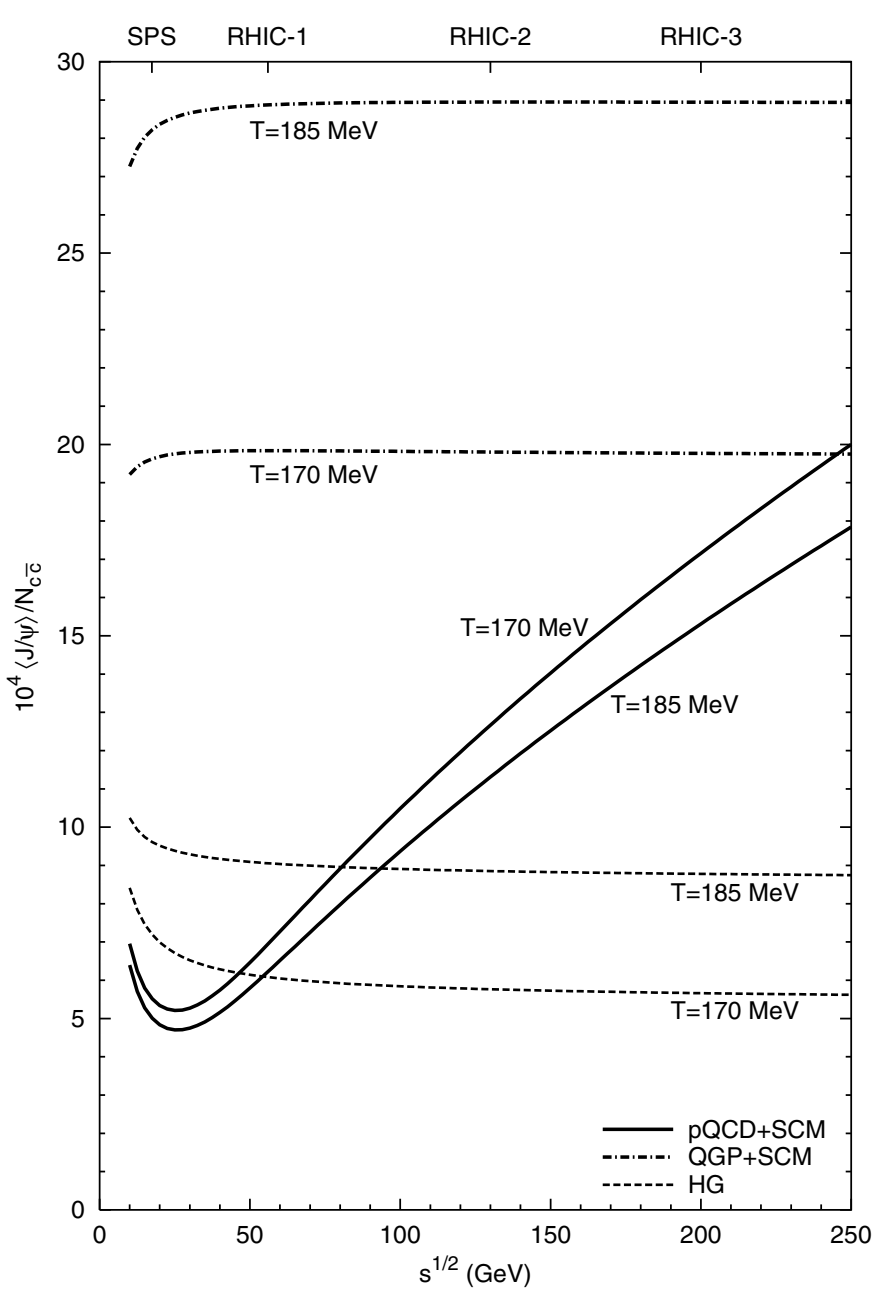

Figure 4. The dependence of the ratio of the $J / \psi$-multiplicity to the multiplicity of cic pairs in $\mathrm{Au}+\mathrm{Au}$ central collisions on the centre-of-mass energy of the nucleon pair. The calculations are done in the framework of three different models: statistical coalescence model with the number of $\mathrm{c} \overline{\mathrm{c}}$ pairs given by perturbative QCD (pQCD+SCM), statistical coalescence model with the number of $c \bar{c}$ pairs given by equilibrium quark-gluon plasma (QGP+SCM) and equilibrium hadron gas model (HG). All the dependences are shown for two different temperatures: $T=170 \mathrm{MeV}$ and $T=185 \mathrm{MeV}$.

\section{3. $J / \psi$ multiplicity ratio to open charm}

The dependence of the $J / \psi$ to $N_{\mathrm{c} \overline{\mathrm{c}}}$ ratio on the collision energy is shown in figure 4. In the HG model and in QGP+SCM, the ratio of the $J / \psi$ to the number of ce pairs is constant at high collision energies. This is because both the $J / \psi$ multiplicity and the number of c $\overline{\mathrm{c}}$ pairs are proportional to the system volume $V$. In contrast, the pQCD+SCM model predicts an essential increase of the ratio by a factor of about 3 from the lowest to the highest RHIC energy.

Results presented in figures 1-4 correspond to the most central $\mathrm{Au}+\mathrm{Au}$ collisions $\left(N_{\mathrm{p}} \approx 2 A\right)$. The dependence of the ratio $R$ on the number of participating nucleons $N_{\mathrm{p}}$ is shown in figure 5. (Note that statistical models are expected to be valid for $N_{\mathrm{p}}>100$.) 


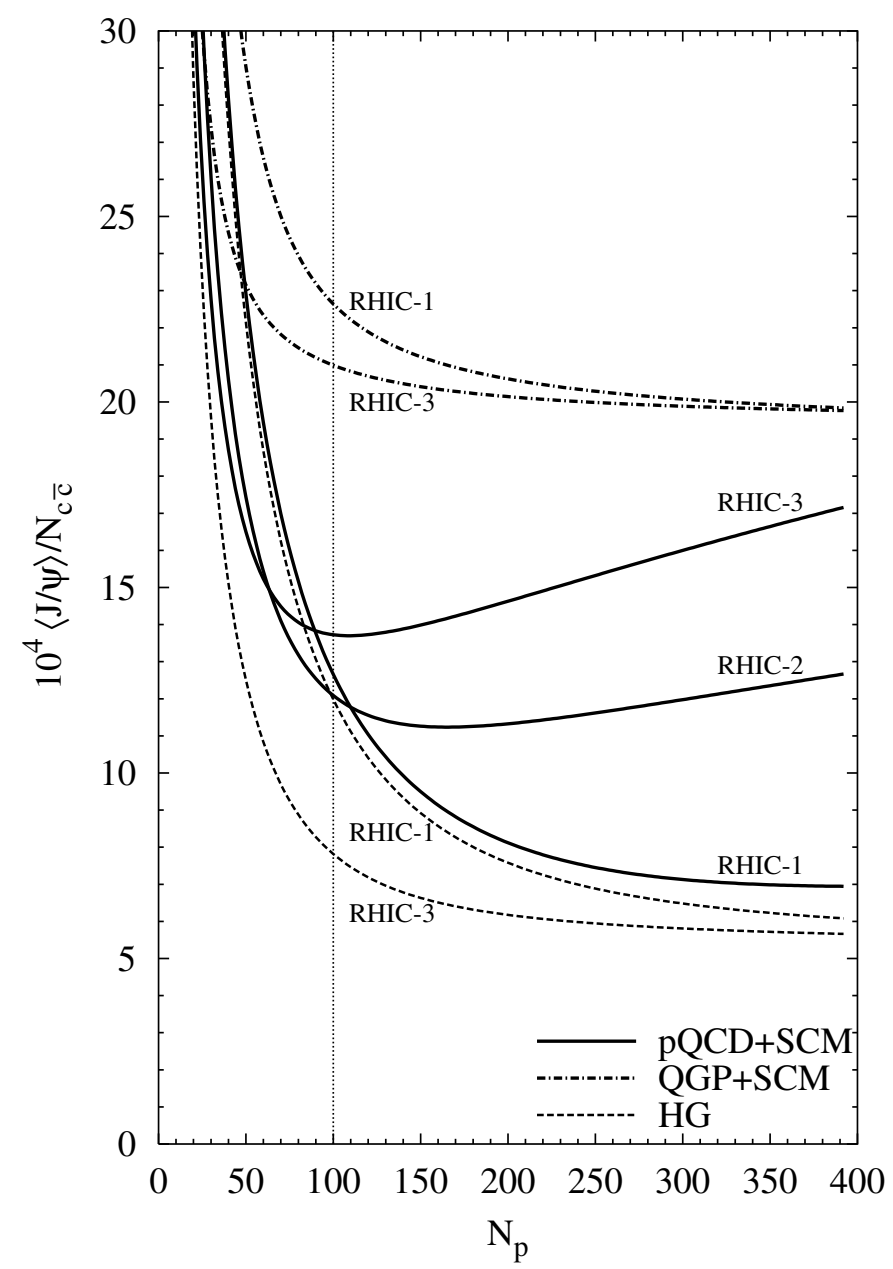

Figure 5. The dependence of the ratio of the $J / \psi$-multiplicity to the multiplicity of ci pairs on the number of nucleon participants $N_{\mathrm{p}}$ for $\mathrm{Au}+\mathrm{Au}$ collisions. The calculations are done in the

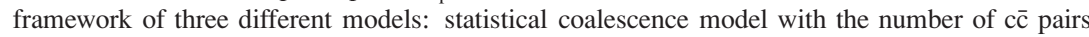

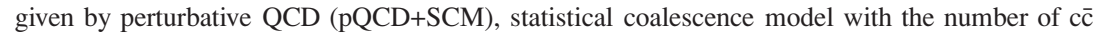
pairs given by equilibrium quark-gluon plasma (QGP+SCM) and equilibrium hadron gas model (HG). In pQCD+SCM case, the dependences are shown for three collision energies per nucleon pair: RHIC-1 $(\sqrt{s}=56 \mathrm{GeV})$, RHIC-2 $(\sqrt{s}=130 \mathrm{GeV})$ and RHIC-3 $(\sqrt{s}=200 \mathrm{GeV})$. For $\mathrm{QGP}+\mathrm{SCM}$ and $\mathrm{HG}$, the results at RHIC-1 and RHIC-3 are presented. The freeze-out temperature was fixed at $T=170 \mathrm{MeV}$. The models are assumed to be valid for $N_{\mathrm{p}} \geqslant 100$, i.e. to the right from the dotted vertical line.

Within pQCD+SCM, one observes both the $J / \psi$ suppression and the $J / \psi$ enhancement

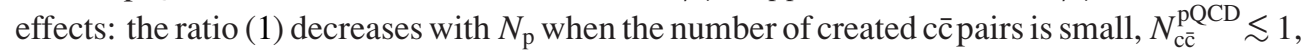
but it begins to increase with $N_{\mathrm{p}}$ when $N_{\mathrm{c} \overline{\mathrm{c}}}^{\mathrm{pQCD}}$ is essentially larger than unity. In the HG model and in QGP+SCM, the suppression is also observed at small $N_{\mathrm{p}}$ corresponding to $N_{\mathrm{c} \bar{c}}^{\mathrm{pQCD}} \lesssim 1$. In contrast to $\mathrm{pQCD}+\mathrm{SCM}$, the models with statistical charm production do not demonstrate $J / \psi$ enhancement: at large $N_{\mathrm{p}}$, which correspond to $N_{\mathrm{c} \overline{\mathrm{c}}}^{\mathrm{pQCD}} \gg 1$, the ratio $R$ tends to a constant value. 


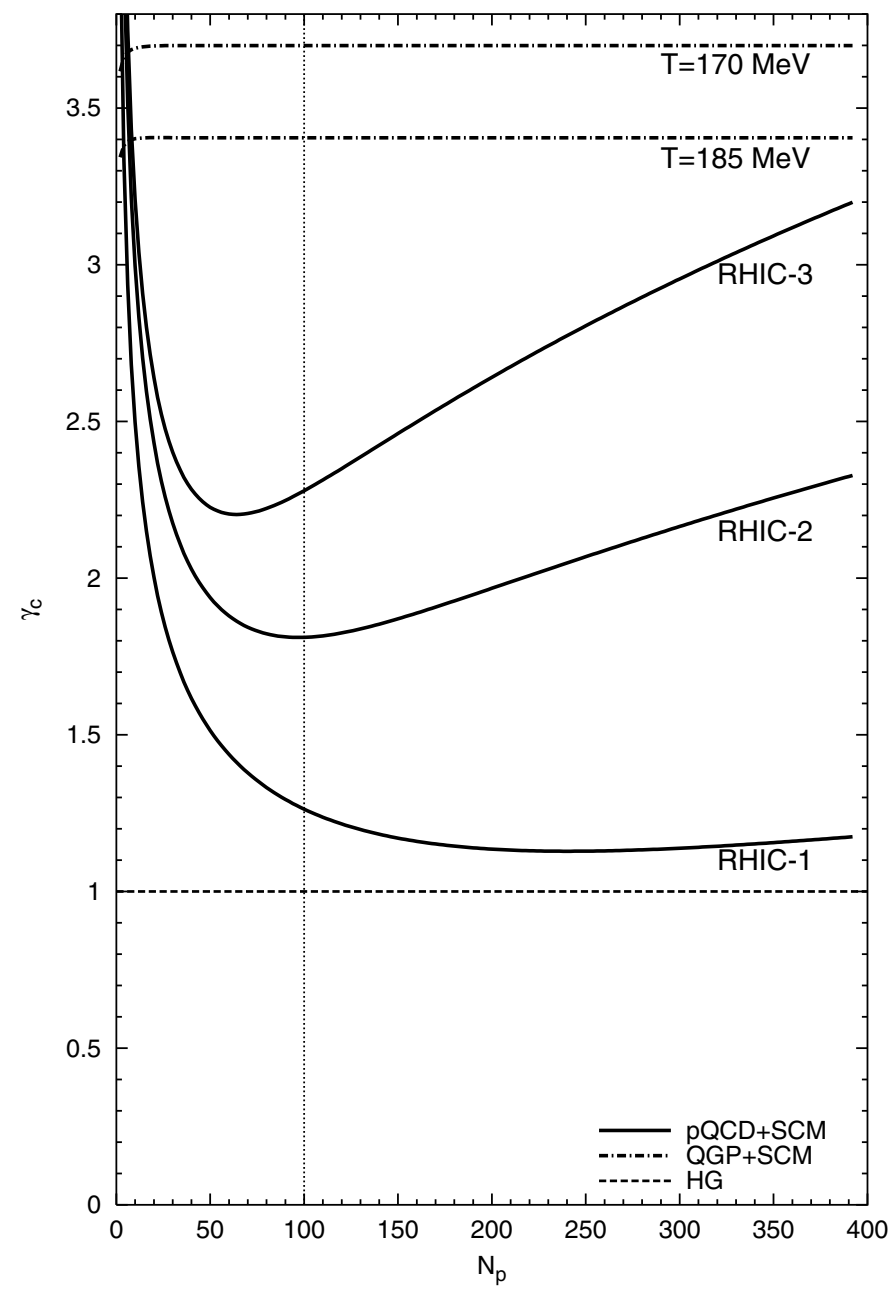

Figure 6. The dependence of the charm enhancement factor $\gamma_{\mathrm{c}}$ on the number of nucleon participants $N_{\mathrm{p}}$ for $\mathrm{Au}+\mathrm{Au}$ collisions. The results are presented for three different models: statistical coalescence model with the number of $c \bar{c}$ pairs given by perturbative QCD (pQCD+SCM), statistical coalescence model with the number of $\mathrm{c} \overline{\mathrm{c}}$ pairs given by equilibrium quark-gluon plasma (QGP+SCM) and equilibrium hadron gas model (HG). In pQCD+SCM case, the dependences are shown for three collision energies per nucleon pair: RHIC-1 $(\sqrt{s}=56 \mathrm{GeV})$, RHIC-2 $(\sqrt{s}=130 \mathrm{GeV})$ and RHIC-3 $(\sqrt{s}=200 \mathrm{GeV})$. The freeze-out temperature was fixed at $T=170 \mathrm{MeV}$. For QGP+SCM, the value of $\gamma_{\mathrm{c}}$ is shown at two values of freeze-out temperature $T=170 \mathrm{MeV}$ and $T=185 \mathrm{MeV}$ (the dependence on the collision energy is negligible). For the equilibrium hadron gas model $(\mathrm{HG}) \gamma_{\mathrm{c}} \equiv 1$ by the definition. The models are assumed to be valid for $N_{\mathrm{p}} \geqslant 100$, i.e. to the right from the dotted vertical line.

\subsection{Centrality dependence of $\gamma_{\mathrm{c}}$}

The dependence of charm enhancement factor $\gamma_{\mathrm{c}}$ on the number of participating nucleons $N_{\mathrm{p}}$ is shown in figure 6. In the case of pQCD+SCM model, the behaviour is similar to that of

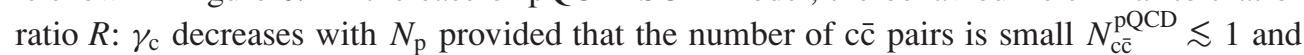
increases at large $N_{\mathrm{c} \bar{c}}^{\mathrm{pQCD}}$. In contrast, the QGP+SCM model leads to a nearly constant value of $\gamma_{c}$, which can easily be explained comparing equations (15) and (22) and neglecting the 
small term $\gamma_{\mathrm{c}}^{2} N_{\mathrm{H}}$. From the same reason the dependence of $\gamma_{\mathrm{c}}$ on $\sqrt{s}$ is also negligible. (Let us remind that in the case of HG model, $\gamma_{\mathrm{c}} \equiv 1$ by the definition).

\subsection{Discussion}

The results presented in figures 4-6 can be easily studied analytically in the limiting cases. For $N_{\mathrm{c} \overline{\mathrm{c}}} \gg 1$ one finds from equation (15)

$$
R=\frac{\langle J / \psi\rangle}{N_{\mathrm{c} \overline{\mathrm{c}}}} \sim \gamma_{\mathrm{c}} \approx 2 N_{\mathrm{c} \overline{\mathrm{c}}} / N_{\mathrm{O}} \sim \frac{N_{\mathrm{c} \overline{\mathrm{c}}}}{V} \sim \frac{N_{\mathrm{c} \overline{\mathrm{c}}}}{\langle\pi\rangle}
$$

where $\langle\pi\rangle$ is the total pion multiplicity. Therefore, in the limiting case $N_{\mathrm{c} \overline{\mathrm{c}}} \gg 1$, the ratio of the $J / \psi$ to the open charm (or a probability to form the charmonium states) is proportional to the factor $\gamma_{\mathrm{c}}$, which in its turn is proportional to the density of ce pairs. In the case of

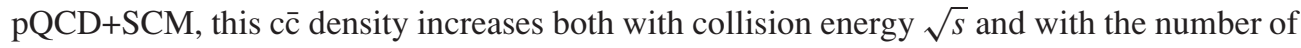
nucleon participants $N_{\mathrm{p}}$ :

(1) the increase (20) of $N_{\mathrm{c} \bar{c}}^{\mathrm{pQCD}}$ with $\sqrt{s}$ is much stronger than the increase of the system volume $V$ or, equivalently, the increase of the total pion multiplicity (11);

(2) the increase of $N_{\mathrm{c} \overline{\mathrm{c}}}^{\mathrm{pQCD}} \sim N_{\mathrm{p}}^{4 / 3}$ is stronger than $V \sim\langle\pi\rangle$ which is approximately proportional to $N_{\mathrm{p}}$.

In the case of thermal charm production (QGP+SCM and HG), the number of ce pairs is proportional to the system volume. Therefore the ratio $R$ becomes constant when $N_{\mathrm{c} \overline{\mathrm{c}}} \gg 1$.

The behaviour of the $\langle J / \psi\rangle / N_{\mathrm{c} \overline{\mathrm{c}}}$ ratio is rather different when $N_{\mathrm{c} \overline{\mathrm{c}}} \ll 1$ and the canonical suppression factor in equation (15) plays a crucial role. One finds $\gamma_{\mathrm{c}}^{2} \approx 4 N_{\mathrm{c} \bar{c}} / N_{\mathrm{O}}^{2}$ and then $\gamma_{\mathrm{c}} \sim N_{\mathrm{p}}^{-1 / 3}$ for pQCD+SCM. The value of $R$, however, does not depend on $\gamma_{\mathrm{c}}$ :

$$
R=\frac{\langle J / \psi\rangle}{N_{\mathrm{c} \overline{\mathrm{c}}}} \sim \frac{1}{V} \sim \frac{1}{\langle\pi\rangle} .
$$

The ratio (1) decreases with both the collision energy (approximately like $(\sqrt{s})^{1 / 2}$ ) and the number of nucleon participants (approximately like $N_{\mathrm{p}}^{-1}$ ) and does not depend on the charm production mechanism. This behaviour is similar to the standard picture of the $J / \psi$ suppression.

The $J / \psi$ suppression in pQCD+SCM occurs at the SPS energy. This energy is still 'low' as $N_{\mathrm{c} \bar{c}}^{\mathrm{pQCD}}<1$ even in the most central $\mathrm{Pb}+\mathrm{Pb}$ collisions. The $J / \psi$ suppression (i.e. the decrease of the $J / \psi / N_{\mathrm{c} \overline{\mathrm{c}}}^{\mathrm{pQCD}}$ ratio) with $N_{\mathrm{p}}$ and/or $\sqrt{s}$ continues up to the RHIC energies for the peripheral collisions with small number of nucleon participants. This changes into the $J / \psi$ enhancement when both $N_{\mathrm{p}}$ and $\sqrt{s}$ are 'large'. Therefore, if the co production is described by the pQCD, the statistical coalescence model predicts the decrease of the ratio $R$ (1) with $\sqrt{s}$ and/or $N_{\mathrm{p}}$ (the $J / \psi$ suppression) when $N_{\mathrm{c} \bar{c}}^{\mathrm{pQCD}} \ll 1$. However, this ratio increases with $\sqrt{s}$ and/or $N_{\mathrm{p}}$ (the $J / \psi$ enhancement) when $N_{\mathrm{c} \overline{\mathrm{c}}}^{\mathrm{pQCD}} \gg 1$. These limiting behaviours are smoothly connected in the intermediate region of $N_{\mathrm{c} \overline{\mathrm{c}}}^{\mathrm{pQCD}} \simeq 1$.

Other models for $J / \psi$ production via coalescence of $\mathrm{c}$ and $\overline{\mathrm{c}}$ at the hadronization stage [7, 8] give similar qualitative result: the $J / \psi$ enhancement for central collisions at high energies. Quantitative predictions, however, are rather different. In [8] $\langle J / \psi\rangle=$ (2.0-3.0) $\times 10^{-1}$ per central $\mathrm{Au}+\mathrm{Au}$ event at $\sqrt{s}=200 \mathrm{GeV}$, whereas our result in pQCD+SCM is $\langle J / \psi\rangle \simeq 1.0 \times 10^{-2}$ (note that the $N_{\mathrm{c} \overline{\mathrm{c}}}$ estimates are very close: approximately 10-11 c c pairs per event). The predictions of the microscopical coalescence model of [7] can be compared with our results at $\sqrt{s}=130 \mathrm{GeV}$. They are rather close for $J / \psi$ to negative hadron ratio: $\langle J / \psi\rangle /\left\langle h^{-}\right\rangle=1.6 \times 10^{-6}[7]$ and $\langle J / \psi\rangle /\left\langle h^{-}\right\rangle \simeq 2 \times 10^{-6}$ in the pQCD+SCM. 
However, the total charm production is rather different: $N_{\mathrm{c} \overline{\mathrm{c}}} \approx 12$ in [7] and $N_{\mathrm{c} \overline{\mathrm{c}}} \approx 5$ in our pQCD estimation.

\section{Conclusion}

The statistical production of $J / \psi$ mesons in $\mathrm{Au}+\mathrm{Au}$ collisions at RHIC energies has been studied. The obtained results are qualitatively different from the standard picture of the $J / \psi$ suppression in A+A collisions [1,2] if the average number of produced charmed quark-antiquark pairs per event is large $N_{\mathrm{c} \overline{\mathrm{c}}} \gg 1$. The standard picture leads to monotonic decrease of the ratio $R$ (the ratio of the $J / \psi$ multiplicity to the total number of charm quark-antiquark pairs) with both the collision energy and the number of participating nucleons. We predict another behaviour. The reason for this difference is the completely distinct physical pictures for charmonia formation. The standard picture supposes the charmonia states to be created exclusively in primary nucleon-nucleon collisions at the early stage of the reaction so that all subsequent interactions can only destroy them. We study another possibility: creation of charmed and charmonium particles at the hadronization stage. In our opinion, the most probable scenario of the charmonium production in $\mathrm{A}+\mathrm{A}$ collisions at the RHIC energies is the following (we call it pQCD+SCM): cē pairs are produced exclusively in hard parton collisions at the early stage of $\mathrm{A}+\mathrm{A}$ reaction (pQCD), and these charmed quarks and antiquarks are redistributed in the open and hidden charm hadrons according to the statistical mechanics prescriptions at the hadronization stage (SCM). This model predicts a strong increase of the ratio $R$ with the collision energy or the number of nucleon participants $(J / \psi$ enhancement $)$, provided that the total number of produced $c \bar{c}$ pairs per event is essentially larger than 1 . This condition is fulfilled at the highest RHIC energy $\sqrt{s}=200 \mathrm{GeV}$ already for $N_{\mathrm{p}}=100$ and also at lower RHIC energies for the most central Au+Au collisions $\left(N_{\mathrm{p}} \simeq 2 A\right)$.

To get a feeling of possible effects of the charm creation (annihilation) by secondary parton and/or hadron rescatterings we have considered two extreme models of charm chemical equilibration: the quark-gluon plasma (QGP+SCM) and the hadron gas (HG). Both models predict almost constant ratio $R$ at $N_{\mathrm{c} \overline{\mathrm{c}}} \gg 1$. There is however a quantitative difference: QGP+SCM predicts much larger value of $R$ and much larger value of the total charm than pQCD+SCM and HG models. The predictions of the HG model for the open charm and charmonium production at the upper RHIC energies are below the results of the pQCD+SCM and QGP+SCM.

Our predictions for the behaviour of $R$ at $N_{\mathrm{c} \overline{\mathrm{c}}} \gg 1(\mathrm{~J} / \psi$ enhancement $)$ in the pQCD+SCM are in a qualitative agreement with other coalescence models [7, 8], but quantitatively the results are rather different.

We conclude that $\mathrm{Au}+\mathrm{Au}$ collision experiments at RHIC offer a possibility to disentangle various models of the open charm and charmonia production. Even those models that give very similar results at SPS energies become quantitatively and even qualitatively different at RHIC. Measurements of the $J / \psi$ productions combined with the open charm measurements are needed to give a definite answer about the dominant mechanisms of hidden and open charm production in ultrarelativistic collisions of heavy nuclei.

\section{Acknowledgments}

The authors are thankful to F Becattini, P Braun-Munzinger, K A Bugaev, M Gaździcki, L Gerland, I N Mishustin, G C Nayak, K Redlich and J Stachel for comments and discussions. We acknowledge the financial support of Alexander von Humboldt Foundation, Germany. 
The research described in this publication was made possible in part by award no UP1-2119 of the US Civilian Research and Development Foundation for the Independent States of the Former Soviet Union (CRDF). This manuscript has been authorized under contract no DE-AC02-98H10886 with the US Department of Energy.

\section{References}

[1] Matsui T and Satz H 1986 Phys. Lett. B 178416

[2] Satz H 2000 Rep. Prog. Phys. 631511 (hep-ph/0007069)

[3] Gaździcki M and Gorenstein M I 1999 Phys. Rev. Lett. 834009 (hep-ph/9905515)

[4] Kabana S 2001 J. Phys. G: Nucl. Part. Phys. 27497 (hep-ph/0010228, hep-ph/0010246)

[5] Braun-Munzinger P and Stachel J 2000 Phys. Lett. B 490196 (nucl-th/0007059)

[6] Gorenstein M I, Kostyuk A P, Stöcker H and Greiner W 2001 Phys. Lett. B 509277 (hep-ph/0010148) Gorenstein M I, Kostyuk A P, Stöcker H and Greiner W 2001 J. Phys. G: Nucl. Part. Phys. 27 L47 (hep-ph/0012015)

[7] Csizmadia P and Lévai P 2000 Preprint hep-ph/0008195

Lévai P, Biró T S, Csizmadia P, Csörgö T and Zimányi J 2001 J. Phys. G: Nucl. Part. Phys. 27703 (nucl-th/0011023)

[8] Thews R L, Schroedter M and Rafelski J 2001 Phys. Rev. C 63054905 (hep-ph/0007323)

Thews R L, Schroedter M and Rafelski J 2001 J. Phys. G: Nucl. Part. Phys. 27715 (hep-ph/0009090)

[9] Gavai R, Kharzeev D, Satz H, Schuler G A, Sridhar K and Vogt R 1995 Int. J. Mod. Phys. A 103043 (hep-ph/9502270)

[10] Braun-Munzinger P, Heppe I and Stachel J 1999 Phys. Lett. B 46515 (nucl-th/9903010) Becattini F, Cleymans J, Keranen A, Suhonen E and Redlich K 2001 Phys. Rev. C 64024901 (hep-ph/0002267) Yen G D and Gorenstein M I 1999 Phys. Rev. C 592788 (nucl-th/9808012)

[11] Gonin M et al 1997 PRINT-97-208 Presented at 3rd Int. Conf. on Physics and Astrophysics of Quark Gluon Plasma (ICPAQGP 97) (Jaipur, India, 17-21 Mar. 1997)

[12] Sorge H, Shuryak E and Zahed I 1997 Phys. Rev. Lett. 792775 (hep-ph/9705329)

[13] Redlich K and Turko L 1980 Z. Phys. C 5201

Rafelski J and Danos M 1980 Phys. Lett. B 97279

Cleymans J, Redlich K and Suhonen E 1991 Z. Phys. C 51137

Gorenstein M I, Gażdzicki M and Greiner W 2000 Phys. Lett. B 48360 (hep-ph/0001112)

[14] Particle Data Group 2000 Eur. Phys. J. C 151

[15] Gażdzicki M 2000 Private communication

[16] Gażdzicki M 1999 Phys. Rev. C 60054903 (hep-ph/9809412)

[17] Rafelski J 1991 Phys. Lett. B 262333

[18] Combridge B L 1979 Nucl. Phys. B 151429

[19] McGaughey P L, Quack E, Ruuskanen P V, Vogt R and Wang X 1995 Int. J. Mod. Phys. A 102999 (hep-ph/9411438)

[20] Gluck M, Reya E and Vogt A 1992 Z. Phys. C 53127

[21] Eskola K J, Kajantie K and Lindfors J 1989 Nucl. Phys. B 32337

[22] Gorenstein M I, Kostyuk A P, Stöcker H and Greiner W 2001 Preprint hep-ph/0104071

[23] Kostyuk A P, Gorenstein M I and Greiner W 2001 Phys. Lett. B 519207 (arXiv:hep-ph/0103057) 